\title{
La comunicación de la administración pública en las redes sociales: Ios casos de las ciudades de Buenos Aires y Bolonia
}

Mariano Ure ${ }^{1}$

Recibido: 2015-03-09

Enviado a pares: 2015-03-09
Aprobado por pares: 2015-04-17

Aceptado: 2015-05-18

DOI: $10.5294 /$ pacla.2016.19.1.10

Para citar este artículo / to reference this article / para citar este artigo

Ure, M. (Marzo de 2016). La comunicación de la administración pública en las redes sociales: Ios casos de las ciudades de Buenos Aires y Bolonia. Palabra Clave, 19(1), 240-270. DOI: 10.5294/pacla.2016.19.1.10

\section{Resumen}

El artículo profundiza en los modelos de gestión, la propuesta comunicativa y las estrategias de las administraciones públicas para involucrarse con los ciudadanos en las redes sociales, a más de un lustro de la incorporación de los recursos de la web 2.0 en la comunicación oficial de las instituciones de gobierno, a través del estudio de caso comparado de dos entes locales urbanos, Buenos Aires (Argentina) y Bolonia (Italia). Desde una perspectiva centrada en los paradigmas opuestos "conversacional-unidireccional” y "abierto-cerrado", la investigación recoge los criterios de publicación utilizados por los responsables de las cuentas oficiales de cada una de las ciudades, así como también la gramática de los mensajes y los sujetos y temas que se conectan en ellos. A su vez, analiza los contenidos de las publicaciones de las cuentas institucionales de ambas ciudades en Twitter, Facebook y YouTube durante un período de seis semanas entre los meses de octubre y diciembre de 2014, clasificándolas en categorías elaboradas con el fin de mostrar el nivel de reconocimiento y empoderamiento de los

1 Universidad Católica Argentina, Argentina. mariano_ure@uca.edu.ar 
usuarios en calidad de ciudadanos. La diversidad de las propuestas comunicativas observada entre los casos estudiados pone en discusión la definición acerca de las "buenas prácticas sociales", y plantea la necesidad de ahondar en los márgenes reales que poseen las administraciones públicas para el aprovechamiento de las posibilidades de interacción que ofrecen las plataformas interactivas.

\section{Palabras clave}

Administración pública, redes sociales, usuarios-ciudadanos, conversación, servicio (Fuente: Tesauro de la Unesco). 


\section{The Communication of Public Administration in Social Networks: The Case of the Cities of Buenos Aires and Bologna}

\section{Abstract}

The article delves into management models, communicative proposal and the strategies of the government to engage with citizens on social networks, more than half a decade of incorporating the resources of Web 2.0 in the official communication from the governmental institutions, through the case study two local urban authorities were compared, Buenos Aires (Argentina) and Bologna (Italy). From a perspective focused on opposing paradigms "conversational-unidirectional" and "open-closed", the research includes the publication criteria used by the responsible official accounts of each of the cities, as well as grammar of the messages and subjects and themes that connect them. In turn, it analyzes the contents of the publications of the institutional accounts of both cities on Twitter, Facebook and YouTube for a period of six weeks between the months of October and December 2014, elaborated and classified in categories in order to show the recognition level and empowerment of users as citizens. The diversity of communicative proposals observed among the cases studied raises questions about the definition of "good social practices" and a need to delve into the actual margins held by public authorities for the exploitation of the possibilities of interaction that interactive platforms offer.

\section{Keywords}

Public administration, social networks, user-citizens, conversation, service (Source: Unesco Thesaurus). 


\section{A comunicação da administração pública nas redes sociais: os casos das cidades de Buenos Aires e Bolonha}

\section{Resumo}

Este artigo aprofunda nos modelos de gestão a proposta comunicativa e as estratégias das administrações públicas para se envolver com os cidadãos nas redes sociais, a mais da metade da incorporação dos recursos da Web 2.0 na comunicação oficial das instituições de governo por meio do estudo de caso comparado de dois entes locais urbanos: Buenos Aires (Argentina) e Bolonha (Itália). De uma perspectiva centrada nos paradigmas opostos "conversacional-unidirecional" e "aberto-fechado", a pesquisa reúne os critérios de publicação utilizados pelos responsáveis das contas oficiais de cada uma das cidades, bem como a gramática das mensagens e os sujeitos e temas que conectam entre eles. Por sua vez, analisa os conteúdos das publicações das contas institucionais de ambas as cidades em Twitter, Facebook e YouTube durante um período de seis semanas entre os meses de outubro e dezembro de 2014 classificando-as em categorias elaboradas com o objetivo de mostrar o nível de reconhecimento e empoderamento dos usuários enquanto cidadãos. A diversidade das propostas comunicativas observada entre os casos estudados põe em discussão a definição sobre as "boas práticas sociais" e apresenta a necessidade de aprofundar nas margens reais que as administrações públicas possuem para o aproveitamento das possibilidades de interação que as plataformas interativas oferecem.

\section{Palavras-chave}

Administração pública, redes sociais, usuários-cidadãos, conversa, serviço (Fonte: Tesauro da Unesco). 


\section{De la experimentación a experiencias consolidadas}

El actual entorno tecnológico y los cambios de tendencia en el consumo de medios fueron obligando a las administraciones públicas a adoptar nuevas modalidades de comunicación para el servicio a los ciudadanos. Las redes sociales, sobre todo a partir de su masificación y sus crecientes tasas de penetración en distintos territorios y regiones, se convirtieron en una herramienta indispensable para lograr mayor cercanía con los ciudadanos. El promedio global de población que usa redes sociales, según datos de enero de 2015 , es del $29 \%$, mientras que el que accede a Internet es del $42 \%$ (We Are Social, 2015). El crecimiento del uso de plataformas interactivas es sostenido en cantidad de usuarios (Pew Research Center, 2013), así como también en la proporción de horas diarias que transcurren en ellas respecto del uso de Internet. Según datos de 2014, mientras el promedio global de horas diarias de internautas que acceden a la red a través de un PC es de 4,4 , la cantidad de horas diarias de acceso a las redes sociales para aquellos que tienen creadas cuentas es de 2,4 (We Are Social, 2015).

Gracias a las redes sociales, las administraciones públicas son capaces de facilitar el acceso a los servicios, promover derechos, brindar transparencia a los actos de gobierno, cultivar la participación en los asuntos comunes, generar comunidad y escuchar a los ciudadanos para la mejora permanente de sus propias acciones (Maultasch de Oliveira y Welch, 2013). La primera etapa en la adopción de estas tecnologías en el sector público consistió en la aplicación de prácticas informales y experimentales (Mergel y Bretschneider, 2013). El objeto de interés en esta etapa consistió en analizar y censar las administraciones que habían creado cuentas oficiales en las redes sociales de mayor difusión. La pregunta por la presencia en la web social parecía adecuada cuando las plataformas interactivas resultaban aún una novedad. Sin embargo, esto ha cambiado: Facebook fue creado en 2004, YouTube en 2005 y Twitter en 2006. De la convulsión inicial por la urgencia de tener presencia en las redes sociales, en un contexto de desconocimiento acerca de cómo integrar exitosamente la gestión de las cuentas con la comunicación de las instituciones públicas y los servicios que le compe- 
ten, se maduró hasta alcanzar en la actualidad una etapa de consolidación de la incorporación de recursos y capacidades, así como también de las estrategias comunicativas implementadas. Un número siempre mayor de administraciones públicas poseen equipos de trabajo integrados por expertos que conocen en detalle las dinámicas que caracterizan a las redes sociales, sus lenguajes y posibilidades de interacción con los usuarios. Ya lejos de la improvisación, los funcionarios responsables de la gestión de la comunicación en la web social tienden a realizar periódicamente un monitoreo de lo que los usuarios comentan sobre diferentes asuntos que involucran a la administración pública y utilizan herramientas estadísticas para evaluar el impacto de las publicaciones.

La introducción de guías claras y la publicación de la estrategia oficial de la organización en los medios sociales constituyen el grado más avanzado de la apuesta por las prácticas innovadoras de relación con los ciudadanos (Mergel y Bretschneider, 2013). Distintas administraciones públicas desarrollaron documentos en los que se establecen las pautas de uso de las cuentas institucionales, como es el caso en España de la Generalidad de Cataluña y del Gobierno Vasco, que en 2010 y 2011, respectivamente, formalizaron una Guía de uso y estilo en redes sociales aplicable a su propia jurisdicción. Por su parte, organismos públicos nacionales de distintos países elaboraron documentos que alientan la incorporación de redes sociales en los distintos niveles del sector público y ofrecen lineamientos de buenas prácticas, como el Vademecum pubblica amministrazione e social media del Ministerio para la Administración P y la Innovación de Italia (2011), y el Manual de orientación para participar en redes sociales de la Secretaría de Comunicación Social de Brasil (2013).

En esta etapa de consolidación de la experiencia en la gestión de redes sociales, es apropiado indagar en el modo en el que las administraciones públicas se están comunicando. Esto cambia el enfoque mayoritario desarrollado hasta ahora, centrado en el análisis de la presencia en la web social y en el grado de aprovechamiento de las posibilidades de interacción, hacia la especificación del tipo de contrato comunicativo que las mismas administraciones están ofreciendo a los usuarios y seguidores de sus cuentas. Por 
contrato comunicativo entendemos, aquí, la propuesta comunicativa de la administración pública, a partir de la cual los ciudadanos pueden articular expectativas realistas de intercambio con ella y acerca del tipo de contenidos a los cuales tendrán acceso.

A diferencia de las privadas, las instituciones públicas democráticas deben estar al servicio de los ciudadanos, actuar de manera abierta, transparente, inclusiva, y empoderar a la comunidad. La pregunta es, entonces, bajo qué lógica son empleadas las redes sociales en el sector público y qué pueden hacer los usuarios-ciudadanos con los contenidos que se publican. Esto lleva a preguntarse por el involucramiento y la apertura a la conversación, que requiere reconocimiento y la simultaneidad de decir y escuchar (Ure y Parselis, 2013), y se manifiesta en la facilitación de la expresión de los ciudadanos y en la valoración y utilización de sus recursos en el abordaje de asuntos de interés para la comunidad. Pero, además, por los mecanismos contrarios, que blindan la identidad del ente público, a través de mensajes autorreferenciales y de mera promoción de sus acciones, o incluso de la imagen de sus funcionarios.

El objetivo general de la investigación es contribuir a delinear las características del contrato comunicativo de las administraciones públicas en las redes sociales, a través de un estudio de caso comparado de las cuentas institucionales oficiales de Twitter, Facebook y YouTube del Gobierno de la Ciudad de Buenos Aires (Argentina) y de la Comuna de Bolonia, capital de la Ciudad Metropolitana de Bolonia (Italia). Para ello, se indaga en el modelo de gestión de la comunicación 2.0 a través del testimonio de los funcionarios con responsabilidades en el área, se observan las estrategias y acciones comunicativas conversacionales en las cuentas, y se clasifican los contenidos de las publicaciones registradas en un periodo de seis semanas de actividad. La selección de las ciudades, una latinoamericana y otra europea, responde a la intención de indagar bajo la misma metodología dos jurisdicciones con diferentes niveles de desarrollo institucional y trayectoria democrática en lo que respecta a la separación entre la administración pública y los intereses político-partidarios. 


\section{La administración pública en las redes sociales}

\section{Incorporación y desaprovechamiento de las posibilidades de las tecnologías sociales}

Según estudios realizados en distintos países, cada vez son más las administraciones públicas que utilizan las redes sociales, en un proceso sostenido de incorporación de las nuevas tecnologías de la información para mejorar el servicio a los ciudadanos (Sixto García, 2012; Mergel, 2013; Arata, 2014; Cippec, 2014). Las redes sociales "están contribuyendo positivamente a las metas de conseguir una administración pública mejor, más sencilla, colaborativa y conectada” (Osimo y Punie, 2008, p. 77). Estas plataformas permiten generar innovaciones en la administración pública, sobre todo en la provisión de servicios y en las dinámicas democráticas. Si bien todavía alcanzan a un porcentaje limitado de la población, "este tipo de instrumentos puede facilitar la interacción con grupos sociales que tradicionalmente son reticentes al contacto con las entidades públicas de cualquier país, sobre todo los más jóvenes, pero también otros como extranjeros no residentes o emigrantes residentes en otros países" (Criado, 2011, p. 3). A su vez, permiten alcanzar a los distintos tipos de usuarios: a los expertos en el lenguaje de las redes, a los que participan agregando comentarios a las publicaciones, $y$ a los que simplemente se benefician del conocimiento suministrado por otros usuarios leyendo sus publicaciones (Osimo y Punie, 2008). En efecto, las redes sociales pueden considerarse como un tipo de innovación tecnológica "disruptiva” (Criado y Rojas Martín, 2013), ligada a la cultura 2.0 de la que emanan. Gracias a las redes sociales resulta más fácil buscar información y acceder a recursos de conocimiento, publicar e intercambiar opiniones y experiencias. Para las administraciones públicas esto implica considerar que los ciudadanos son potenciales agentes activos de producción de contenidos, que la innovación pública se encuentra fuera de los límites formales de la organización y que las actividades entre organizaciones e individuos particulares está de manera creciente desintermediada (Criado y Rojas Martín, 2013).

Sin embargo, no basta con la adopción de tecnologías interactivas: es necesario que el sector público cambie la relación con los usuarios y que 
esta sea "más abierta y transparente" (Osimo y Punie 2008, p. 77), además de inclusiva, ya que "tanto la baja participación como la participación únicamente de usuarios 'elitistas' son dos situaciones poco deseables” (Osimo y Punie, 2008, p. 75). La mayor crítica al uso actual de las redes sociales es la falta de disposición de las administraciones al diálogo con el ciudadano y el desaprovechamiento de los recursos que ofrecen para iniciar un intercambio abierto y enriquecedor que permita pasar del e-government al we-government. La interacción es escasa, se privilegia la simple presencia y las cuentas están todavía pensadas para la autopromoción y no tanto para la participación y la colaboración ciudadana con el gobierno (Pereyra Caramé, 2012).

El sector público subestimó la centralidad y el protagonismo que posee el ciudadano en las democracias modernas. Hasta el momento, "no logró garantizarles servicios a medida y usó el canal más idóneo, la web, casi exclusivamente para promocionarse a sí misma en un delirio de narcisismo que produjo más daños que ventajas" (Cogo, 2012, p. 14). Sin embargo, las redes sociales son un fenómeno ineludible y las entidades de gobierno deben acostumbrarse a un entorno en el que la información y el conocimiento son también creados por las personas fuera de las fronteras de la organización, a través de medios colaborativos, por lo que las redes sociales digitales no son meros medios de comunicación, sino que se convierten en mecanismos orientados a la integración de los ciudadanos en las actividades de las instancias públicas (Criado, 2011).

Ese es, sin embargo, el principal motivo y uso que se hace de las plataformas interactivas, por lo cual no parece que, por el momento, las herramientas de la web 2.0 estén conduciendo a una significativa revolución en la relación entre el gobierno y los ciudadanos, sino que solo suponen un paso adelante para "proveer información y servicios a audiencias externas" (Bonsón, Torres, Royo y Flores, 2012, p. 131).

Una investigación sobre los usos primordiales de Facebook y Twitter en las comunas, provincias y regiones italianas conducida en 2010 da cuenta del "uso asocial de los medios sociales" (Arata, 2014, p. 325). En su conjunto, los entes italianos muestran un grado limitado de competencias respecto a gramáticas y prácticas de las redes sociales, y las emplean como 
meras extensiones digitales de la oficina de prensa. Lo confirman "la no valorización de las funciones de escucha y diálogo que ofrecen Facebook y Twitter, por ejemplo menciones, retweet, contenidos compartidos, que permitirían salir de la lógica broadcast poniendo en valor las contribuciones de los ciudadanos" (Arata, 2014, p. 325). La investigación observa, también, cierta superposición entre la comunicación institucional y la comunicación política. En ocasiones los espacios institucionales se pueblan de batallas personales de los funcionarios electos, que carecen de relevancia desde el punto de vista institucional.

Una conclusión similar plantea un estudio realizado entre septiembre de 2010 y marzo de 2011, en el que se entrevistó, a través de cuestionarios digitales, a los responsables de la comunicación de las comunas italianas de más de 15000 habitantes (Montanari, Pattaro y Scapolan, 2013). Las redes sociales son utilizadas por las comunas mayormente para proveer información a la ciudadanía y, en menor medida, para recoger informaciones de los ciudadanos y comenzar discusiones y debates. Los entes locales reconocen en estos medios instrumentos capaces de generar innovaciones que pueden mejorar algunos procesos importantes de gobierno, como la oferta de servicios, la planificación estratégica de las actividades, la definición y la evaluación de las políticas públicas. Los principales motivos por los que las comunas invierten en medios sociales son: aumentar las interacciones con los ciudadanos, alcanzar franjas más jóvenes de la población y mejorar la inmediatez de las comunicaciones con los ciudadanos y la reducción de los costos de la comunicación; introducir nuevos servicios, en cambio, está menos difundido. Sin embargo, el estudio muestra que en la mayor parte de los casos las acciones de las comunas en las redes responden casi exclusivamente a procesos de comunicación unidireccional.

Más que a la generación de una red de participación y colaboración, los usos de Facebook por parte de las administraciones públicas están claramente orientados hacia la proyección comunicativa de una imagen institucional (Criado y Rojas Martín, 2013). Un análisis de las páginas activas de Facebook de 14 ayuntamientos españoles, realizado en 2011, muestra que la utilidad de la información ofrecida es "de refuerzo respecto a lo que 
distribuyen a través de otros canales de comunicación" (Lizette Martínez, 2011 , p. 7). La mayoría de los ayuntamientos muestra una interactividad media o baja, y es frecuente que el ciudadano les dirija preguntas y que no encuentre respuestas de los entes. Si bien los ayuntamientos realizaron esfuerzos para adaptarse a los medios 2.0, la realidad muestra que son utilizados como espacios de anuncios, lo que refuerza el modelo unidireccional de "uno-a-muchos" (Lizette Martínez, 2011).

En esa misma línea se encuentra la investigación sobre la incorporación de las redes sociales en 22 instituciones de gobierno en Galicia, España. Las instituciones gallegas están presentes activamente en las redes, pero son elegidas como instrumentos para difundir información antes que para participar realmente en ellas (Sixto García, 2012). Según el estudio, todas las instituciones justifican su presencia en las redes por "la capacidad que ofrecen para que los usuarios difundan o expandan la información a otros contactos, de modo que los usuarios no sólo actuarían como receptores de contenidos, sino también como distribuidores e incluso creadores" (Sixto García, 2012, p. 362). Esto hace que las potencialidades de las redes estén poco explotadas y que la comunicación de las instituciones en esa región de España reproduzca modelos propios del sistema 1.0.

El uso de Twitter de los ayuntamientos catalanes también prioriza la difusión unidireccional de información. Casi todos los responsables de la comunicación de los entes encuestados están de acuerdo en que el objetivo de utilizar esta red social es mantener informados a los ciudadanos y hacer que las actividades del gobierno local sean más visibles (Balcells, Padró-Solanet y Serrano, 2013). Sin embargo, aparece mayor desacuerdo acerca de la posibilidad de relacionarse con los ciudadanos. En efecto, fuera de informar a los ciudadanos, otras posibles funciones de Twitter parecen menos prioritarias para los funcionarios a cargo de la comunicación, como la construcción de redes ciudadanas, el fortalecimiento de las asociaciones locales, la posibilidad de que los ciudadanos puedan expresar sus demandas o la mejora en la capacidad de respuesta del gobierno local. Esto se comprende ya que "apostar por una cuenta interactiva es, de hecho, una decisión estratégica y, sin duda, requiere de un mayor esfuerzo organiza- 
tivo" (Balcells, Padró-Solanet y Serrano, 2013, p. 75). De hecho, las cuentas más activas suelen estar gestionadas por responsables de comunicación que creen en el potencial de Twitter para involucrar a los ciudadanos, tanto respecto de la canalización de las demandas ciudadanas como de la mejora de la capacidad de respuesta de los gobiernos locales.

A abonar esta crítica asiste la investigación sobre las páginas oficiales de Facebook de 12 de las 15 ciudades alemanas con mayor cantidad de habitantes, conducida en abril de 2012, que concluye que el comportamiento comunicativo online de los gobiernos locales está basado en la diseminación de información en un modo tradicional, sin adaptar sus hábitos comunicacionales a las características de las redes sociales (Hofmann, Beverungen, Räckers y Becker, p. 2013). También, el caso de estudio de los posts en las cuentas de tres ciudades norteamericanas, Seattle, Louisville y Chicago, realizado en 2011, las cuales muestran mayormente una estrategia comunicativa denominada push, que se caracteriza por usar las redes para proveer información a los usuarios, en lugar de una estrategia de networking con el público, que es interactiva y provee una buena cantidad de posts con información adicional requerida por los usuarios (Mossberger, Wu y Crawford, 2013).

\section{Las ciudades de Buenos Aires y Bolonia en las redes sociales}

El tiempo de conexión a Internet, la penetración de las redes sociales y la preferencia de los usuarios por la actividad social en la web muestran un crecimiento constante. En Argentina, la actividad en las redes sociales es el uso más extendido que se le da a Internet. Según la Encuesta Nacional de Consumos Culturales y Entorno Digital (Secretaría de Cultura de la Nación, 2013), el $57 \%$ de los usuarios las utilizan, y un $46 \%$ lo hace con frecuencia. Facebook es la red preferida de los argentinos y YouTube aparece en segundo lugar. El $55 \%$ de la población posee un perfil en Facebook y el $13 \%$ en Twitter. De acuerdo con datos de ComScore, Facebook es la red social con mayor cantidad de visitantes únicos en Argentina, con 15,5 millones, mientras que Twitter ocupa el tercer lugar, con cuatro millones. En la distribución del tiempo consumido en redes sociales, Facebook acapara el $96 \%$ y Twitter, el $2 \%$ (ComScore, 2014). 
También en Italia Facebook es la red más extendida. El 70 \% de las personas que poseen acceso a Internet abrió una cuenta en Facebook, lo que corresponde al $44 \%$ de la población del país (Censis/Ucsi, 2013). YouTube alcanza el $61 \%$ de los internautas (39\% de la población), mientras que el $15 \%$ utiliza Twitter (el $9 \%$ de los italianos). El promedio de horas transcurridas por los usuarios italianos en redes sociales, en el mes agosto de 2013, fue de 9,5, mientras que para los argentinos fue de 9,1, muy por encima del promedio global de 5,4 (ComScore, 2013).

La Ciudad de Buenos Aires (BA) constituye una jurisdicción administrativa autónoma integrada por tres poderes locales, ejecutivo, legislativo y judicial, en la que residen cerca de tres millones de habitantes. Para esta investigación se considera el uso de redes sociales del poder ejecutivo local: el Gobierno de la Ciudad de Buenos Aires. La ciudad de Bolonia (BO) es la capital de la Ciudad Metropolitana de Bolonia, con poco más de un millón de habitantes. Ambas administraciones están presentes activamente en las redes sociales y poseen una trayectoria consolidada a lo largo de más de un lustro.

Desde el home del sitio web institucional del Gobierno de la Ciudad de Buenos Aires (http://www.buenosaires.gob.ar) es posible acceder a las cuentas oficiales de Twitter y Facebook, las cuales fueron creadas en el año 2009. La administración también generó cuentas en otras redes sociales como YouTube, en 2006; Flickr, en 2009; Google Plus, en 2011, e Instagram, en 2014. Las cuentas de Flickr y Google Plus muestran un escaso nivel de actualización. La comuna de Bolonia permite, en cambio, el acceso a todos sus perfiles de redes sociales a través de íconos en su sitio web institucional (http://www.comune.bologna.it). Las cuentas de Twitter, Facebook y YouTube fueron generadas en el año 2009, mientras que la de Pinterest, en 2011, y la de Instagram y Flickr, en 2012.

Si bien las administraciones públicas de ambas ciudades adoptaron una estrategia similar en lo que respecta al inicio de la actividad en las redes sociales y a las plataformas escogidas para comunicarse con los usuarios-ciudadanos, el volumen de publicaciones y de seguidores varía notablemente. 
Al 31 de diciembre de 2014, la cuenta de Twitter @gcba, perteneciente a BA, lleva acumulados unos 12000 tuits y posee más de 480000 seguidores. La cuenta de BO, @Twiperbole, acumula un mayor número de tuits, poco más de 40000 , pero un valor significativamente menor de seguidores, unos 22 000. La diferencia en la cantidad de seguidores se observa, también, en la página de Facebook. La de BA posee más de 635000 “me gusta”, mientras que la de BO cerca de 13 000. Si bien el canal de BA en YouTube lleva la ventaja de haber sido generado tres años antes que el de BO, la diferencia entre ambos canales es significativa. Mientras el de BA cuenta con 6250 suscriptores y lleva acumuladas más de 51 millones de visualizaciones, el de $\mathrm{BO}$ posee 200 suscriptores y 73000 visualizaciones.

\section{Metodología}

La investigación se desdobla en una parte cualitativa y otra cuantitativa. La primera consiste en la realización de entrevistas en profundidad a los responsables de la política institucional y la gestión de la comunicación en redes sociales de las administraciones públicas locales de Buenos Aires y Bolonia, además de la observación de las estrategias y prácticas de involucramiento con los usuarios-ciudadanos. Cabe aquí distinguir entre involucramiento con y de los usuarios. El primero supone interés del emisor por la situación del interlocutor y la promoción de su participación en asuntos comunes para ambos, los cuales, una vez afrontados, ofrecerían beneficios recíprocos. El segundo, en cambio, se entiende, al estilo del engagement rate de Facebook, como repercusión interactiva de lo publicado por el emisor, sin que esto implique reciprocidad de intercambio.

Las entrevistas se centraron en los siguientes puntos: el modelo de gestión (centralizado con una única cuenta o descentralizado con cuentas temáticas o por áreas), la construcción de la agenda (criterios editoriales: qué se publica, con qué frecuencia, cómo se clasifica la información), la interacción con otros usuarios (cómo se busca el involucramiento de y con los usuarios-ciudadanos), las herramientas de monitoreo de la actividad de los usuarios-ciudadanos y la existencia de normas o protocolos de actuación (imagen gráfica, estilo lingüístico, a quiénes se sigue, a quiénes se responde, quiénes están autorizados a publicar). 
La observación de estrategias y prácticas apunta a especificar las formas en las que las administraciones de ambas ciudades se disponen a la conversación, y a ofrecer espacios a los ciudadanos para que se expresen libremente y para amplificar sus intereses y opiniones. La observación sirve como revisión de las estrategias enunciadas por los funcionarios en las entrevistas. Concretamente, se observan las formas de interacción con los usuarios-ciudadanos (difusión de contenidos de terceros, respuestas, inclusión de hipervínculos, etc.).

La parte cuantitativa consiste en el registro de las publicaciones de las cuentas oficiales de BA y BO en tres redes sociales de gran difusión (tabla 1), según una categoría de contenidos (tabla 2), elaborada a fin de poder caracterizar su propuesta comunicativa a los usuarios-ciudadanos.

\section{Tabla 1 \\ Cuentas institucionales consideradas}

\begin{tabular}{|l|l|l|}
\hline & Buenos Aires & Bolonia \\
\hline Twitter & $@ g c b a$ & $@$ Twiperbole \\
\hline Facebook & https://www.facebook.com/GCBA?ref=ts\&fref=ts & https://www.facebook.com/comunebologna?fref=ts \\
\hline Youtube & https://www.youtube.com/user/GCBA & http://www.youtube.com/user/ComuneDiBologna \\
\hline
\end{tabular}

En esta investigación se considera la cuenta institucional "principal" de cada administración pública local en cada red social, bajo el supuesto de que aun en modelos de gestión descentralizados con cuentas "temáticas" o por áreas (correspondientes a niveles administrativos inferiores), la cuenta "principal" es aquella que carga con el mayor peso simbólico y la que delinea la imagen general de toda la institución.

Dada la ubicación geográfica de los entes locales estudiados y para evitar distorsiones en la comparación que respondan a las particularidades del calendario de cada hemisferio, el periodo de registro es de seis semanas en total en un arco de tres meses. De esta manera, se garantiza la coincidencia de semanas de mayor actividad política e institucional y semanas más cercanas a lo festivo y de vacaciones escolares. Las seis semanas se componen de la siguiente manera: primera y segunda semana de octubre; segunda y tercera semana de noviembre, y tercera y cuarta semana de diciembre de 2014. 
En esta investigación se consideran unidades de registro las piezas textuales independientes, sin revisar el contenido de los enlaces que puedan contener los posts. Estas unidades son: a) tuits, b) posts en Facebook y c) nuevos videos publicados en el canal de YouTube. Cada una de estas piezas textuales se clasifica según el esquema de categorías de contenidos que se describe a continuación:

\section{Tabla 2 \\ Categorías de contenidos y descripción}

\begin{tabular}{|c|c|}
\hline Categoría de contenidos & Descripción \\
\hline Promocionales & $\begin{array}{l}\text { Invitación a la participación en eventos futuros (festivales culturales, encuentros deportivos), con } \\
\text { por lo menos un día de anticipación y con datos de acceso o programa de actividades. }\end{array}$ \\
\hline Servicio & $\begin{array}{l}\text { Orientación al ciudadano para la realización de trámites o reserva de turnos; información de } \\
\text { acceso a programas de gobierno o concursos; información sobre tránsito, obras, estado del tiempo. }\end{array}$ \\
\hline Bien público & $\begin{array}{l}\text { Mensajes orientados a la prevención (salud, seguridad vial, violencia de género) y campañas } \\
\text { solidarias (donación de sangre, dinero para fines benéficos). }\end{array}$ \\
\hline Institucionales & $\begin{array}{l}\text { Difusión de planes y ampliación informativa de normas y medidas vigentes de gobierno, difusión } \\
\text { de derechos y deberes de los ciudadanos. }\end{array}$ \\
\hline Gobierno abierto & $\begin{array}{l}\text { Publicaciones con actividades de gobierno acompañadas de documentos oficiales, acceso a base } \\
\text { de datos o estadísticas. }\end{array}$ \\
\hline Protocolares & $\begin{array}{l}\text { Adhesión a celebraciones internacionales y felicitaciones a grupos profesionales o comunidades } \\
\text { (día del periodista, inmigrantes según su origen). }\end{array}$ \\
\hline Agenda del día & Información de actividades del día o anuncio de eventos cerrados o sin datos de acceso para el público. \\
\hline Prensa & $\begin{array}{l}\text { Información de actividades mientras se están llevando a cabo o una vez consumadas, de gobierno } \\
\text { y funcionarios o de grupos y organizaciones no gubernamentales, que se intentan propagar y a } \\
\text { las que la ciudadanía ya no puede asistir. }\end{array}$ \\
\hline Comunicación política & $\begin{array}{l}\text { Campaña permanente y promoción del responsable del gobierno local a través de los logros } \\
\text { de la gestión o planes de futuro, acompañados de estadísticas que resaltan las mejoras o con } \\
\text { valoraciones positivas. }\end{array}$ \\
\hline Identidad local & $\begin{array}{l}\text { Mensajes con referencia a lo característico de la ciudad para reforzar la identidad local y y } \\
\text { publicidades orientadas a la promoción de la "marca ciudad" (historias, personajes, descripción } \\
\text { de paisajes urbanos, monumentos, gastronomía distintiva, cultura de los barrios). }\end{array}$ \\
\hline $\begin{array}{l}\text { UGC (User-Generated } \\
\text { Content) }\end{array}$ & $\begin{array}{l}\text { Publicación en la cuenta institucional de contenido enviado por los usuarios-ciudadanos (fotos } \\
\text { de la ciudad, etc.). }\end{array}$ \\
\hline Respuestas & $\begin{array}{l}\text { Publicaciones en las que se responden consultas e inquietudes puntuales de los usuarios- } \\
\text { ciudadanos. }\end{array}$ \\
\hline Cortesía & $\begin{array}{l}\text { Saludos circunstanciales o estacionales (buenos días, bienvenida a la primavera), agradecimientos } \\
\text { (usuarios que colaboran en la cobertura de eventos, etc.). }\end{array}$ \\
\hline Crowdsourcing & $\begin{array}{l}\text { Convocatoria a los usuarios-ciudadanos a colaborar a través del envío de información o ideas en } \\
\text { la solución de algún problema común de la ciudad. }\end{array}$ \\
\hline Otros & $\begin{array}{l}\text { Mensajes exhortativos centrados en un valor cívico (cuidado del medioambiente, etc.), } \\
\text { efemérides, preguntas abiertas, campañas de colecta de fondos, experiencias personales de } \\
\text { usuarios-ciudadanos, etc. }\end{array}$ \\
\hline
\end{tabular}

Fuente: elaboración propia. 
Cabe mencionar que varios posts (publicaciones) de las administraciones púbicas consideradas, sobre todo en Facebook, resultan complejos, ya que contienen información perteneciente a distintas categorías de análisis. En estos casos se clasifican los posts en la categoría con la que inicia el mensaje. Por ejemplo, si un mismo mensaje brinda información sobre el clima e incluye una fotografía compartida por un usuario en alguna red social, en ese orden, se lo registra en la categoría de servicio.

El análisis del peso relativo de cada categoría de contenidos en la actividad total del periodo de registro permite una aproximación al tipo de propuesta comunicativa que cada administración pública local realiza a sus ciudadanos a través de las redes sociales consideradas.

\section{Resultados}

\section{Criterios y usos de redes sociales de los equipos de Buenos Aires y Bolonia}

Las cuentas oficiales generales del Gobierno de la Ciudad de Buenos Aires son gestionadas por la Dirección de Nuevos Medios, dependiente de la Secretaría de Medios. Las cuentas temáticas, como la "agenda cultural", por programas, como el "Plan Sarmiento", o por niveles de administración, como el Ministerio de Salud, son gestionadas por equipos diferentes de comunicación. Esto concede cierto margen de descentralización que, sin embargo, como sostiene Julieta Goldman, directora de Nuevos Medios, intenta revertirse: "Debido al crecimiento de las redes ahora tratamos de unificar y no descentralizar, porque si cada una de las cuentas chicas publica contenidos por separado, es más difícil llegar a una audiencia consolidada”. Para la apertura de cuentas oficiales, las distintas áreas del Gobierno deben solicitar una autorización formal a Nuevos Medios.

El modelo de la comuna de Bolonia es aún más centralizado. Las cuentas son gestionadas por el área de Prensa, a cargo de Cristiano Zecchi, que explica que "anteriormente existían cuentas de diferentes área de la administración, pero eso no era conveniente ya que terminaban compitiendo entre sí por los seguidores". Si bien queda abierta la posibilidad de 
crear perfiles para proyectos puntuales, los institucionales están a cargo del equipo de medios sociales del área de Prensa.

Ambas administraciones públicas carecen de normas de actuación en redes sociales que puedan ser consultadas por los ciudadanos. Lo que se publica, en qué momento, con qué periodicidad y con qué lenguaje, está basado en la práctica del ensayo y el error, es decir, en la experiencia adquirida a lo largo de los años de gestión. La Ciudad de Buenos Aires posee un protocolo solo en caso de crisis, mientras que la agenda de publicaciones sigue algunos ejes temáticos de información: cultura, salud, medioambiente. El equipo de medios sociales de BO, integrado por Luca Zanelli y Daniele Tarozzi, va definiendo su agenda con algunos pocos criterios generales y con una fuerte cuota de espontaneidad. Publica un tuit cada 30 minutos y un post en Facebook cada 180 minutos aproximadamente, buscando que uno posea contenido institucional o de servicio y el otro sea más ligero, como un saludo, una pregunta o fotografías.

Si bien BA tiene presencia en varias redes sociales, las más importante es Facebook, seguida de Twitter y YouTube. "De Facebook tomamos el enorme potencial de poder volcar tu propia información, convirtiéndote en tu propio editor", señala Julieta Goldman. El equipo de medios sociales de $\mathrm{BO}$, en cambio, muestra una gran preferencia por Twitter, porque "es más inmediato y transparente", lo que se observa claramente en la diferencia del nivel de actividad en las distintas redes (tabla 3 ).

\section{Tabla 3}

\section{Cantidad total y promedio de posts publicados en el total del periodo}

\begin{tabular}{|c|c|c|c|c|c|c|}
\hline \multirow{2}{*}{} & \multicolumn{3}{|c|}{ Buenos Aires } & \multicolumn{3}{c|}{ Bolonia } \\
\cline { 2 - 4 } & \multirow{2}{*}{$\begin{array}{c}\text { Cantidad de } \\
\text { posts }\end{array}$} & \multicolumn{2}{|c|}{ Promedio posts } & \multirow{2}{*}{$\begin{array}{c}\text { Cantidad de } \\
\text { posts }\end{array}$} & \multicolumn{2}{c|}{ Promedio posts } \\
\cline { 7 - 8 } \cline { 6 - 7 } & Por semana & Por día & Por semana & Por día \\
\hline Twitter & 235 & 39,16 & 5,59 & 974 & 162,33 & 23,19 \\
\hline Facebook & 101 & 16,83 & 2,40 & 224 & 37,33 & 5,33 \\
\hline YouTube & 29 & 4,12 & 0,69 & 1 & 0,14 & 0,02 \\
\hline
\end{tabular}

Fuente: elaboración propia. 
En lo que respecta al involucramiento con los usuarios-ciudadanos, la estrategia de BA se concentra en estar atentos a aquello sobre lo que los usuarios están interesados. "Muchas veces las redes sociales te permiten ver qué te está diciendo la gente, en los mismos comentarios podés detectar temas, o si hay muchas quejas, muchos pedidos sobre algo en particular, ahí encontramos oportunidades de comunicación", explica Goldman. Si bien se leen todos los comentarios en la página oficial de Facebook, "es imposible responderles a todos, pero sí hacer un englobado de temas", agrega. El monitoreo de los usuarios es permanente: "Una persona se encarga de analizar y detectar temas, que pueden ser positivos o negativos. La generación de contenidos muchas veces se desprende de ahí, si no estás instalando agenda de lo que al gobierno le importa y no a la gente. A veces la comunicación tiene que ser de afuera hacia adentro. Ese fue el gran viraje que tuvimos a partir de leer los comentarios, ver las menciones", completa la directora de Nuevos Medios del Gobierno de la Ciudad de Buenos Aires.

Para involucrarse con los usuarios-ciudadanos, el equipo de medios sociales de $\mathrm{BO}$ utiliza el contenido generado por ellos mismos, lo que "posibilita un intercambio directo". Se agregan preguntas abiertas en los posts, o se utilizan estructuras gramaticales que indican que el contenido proviene de terceros, como "[gli amici di \#Iperbole fotografano] [los amigos de \#Iperbole fotografían]", citando el nombre de usuario de la persona que comparte el contenido. En este sentido, Bolonia lanzó en abril de 2014 el proyecto denominado \#MyBologna are You, que consiste en delegar la gestión de la cuenta oficial de Instagram a distintos ciudadanos durante una semana (http:// iperbole2020.comune.bologna.it/blog/mybologna-are-you-il-profilo-instagram-gestito-dai-cittadini/). Debido al éxito de la iniciativa y para potenciar la participación de los usuarios, el equipo de medios sociales de $\mathrm{BO}$ analiza la posibilidad de confiar la gestión de la cuenta institucional de Twitter a un par de usuarios durante los fines de semana.

Los posts en el perfil de Facebook de BA reciben un gran número de comentarios, que en ocasiones se cuentan por cientos, lo que efectivamente obstaculiza una conversación personalizada con los usuarios. De hecho, 
BA no suele responder a las interpelaciones sino solo circunstancialmente. Los retuits son casi exclusivamente de otras cuentas oficiales del Gobierno de la ciudad y se utilizan pocas menciones con arroba, en particular a usuarios institucionales de gobierno o a celebridades que participan de algún evento organizado por BA. Algo semejante ocurre con los hipervínculos, que remiten al sitio web del Gobierno de la ciudad o a otras cuentas de redes sociales oficiales de BA. Las imágenes insertadas en los posts llevan algún logo que identifica a la Ciudad de Buenos Aires, respetando los lineamientos visuales de marca como el color amarillo (el cual por otra parte coincide con el color insignia del PRO, partido al que pertenece el jefe de Gobierno). Estas acciones refuerzan la identidad institucional de la administración pública.

Las cuentas de BO dan lugar a iniciativas y acciones de terceros. Evitan, sin embargo, la promoción de actividades de neta inspiración comercial. Utilizan menciones con arrobas y etiquetas con hashtags en Twitter para participar de la conversación. Muchos de sus tuits son retuits (RT) o tuits modificados (MT) de otros usuarios, tanto institucionales como individuales. Esto lleva a que incluso se realicen publicaciones completas en inglés, cuando son escritas por usuarios extranjeros. Esto sucede, especialmente, cuando los usuarios mencionan previamente a @Twiperbole en sus posts. Del mismo modo, suelen incorporar fotografías tomadas por usuarios, dando crédito a los autores a través de menciones con arrobas y etiquetas con hashtags, sobre las cuales no se aplica ningún logo oficial de BO. Como estrategia de cercanía, acuden al lenguaje de los emoticones tanto en caritas como en letras, populares sobre todo entre usuarios jóvenes. Además, suelen introducir saludos de buenos días y buenas noches. Los fans de la página de Facebook tienden a marcar "me gusta" pero a comentar circunstancialmente los posts. En caso de señalamientos puntuales por parte de los usuarios, como el pedido de profundización de información o de corrección de información errónea, el equipo de medios sociales suele dar una respuesta. Para ellos, utiliza el recurso gramatical de “[...]" con el fin de orientar la comprensión de la información de la publicación, como “[dal web] [desde la web]", "[\#bandi_news] [\#concursos_noticias]", "[save the date] [agenda la fecha]”, “[\#dai_quartieri] [\#desde los barrios]”, entre otros. 


\section{Registro de publicaciones en Twitter, Facebook y YouTube}

Las seis semanas de registro de la actividad de las cuentas consideradas para la investigación, entre los meses de octubre y diciembre de 2014, muestran que Twitter es la red más utilizada, seguida de Facebook y YouTube (tabla 3). La cuenta de $\mathrm{BO}$ en Twitter posee casi 21 veces menos seguidores, pero un nivel de actividad tres veces superior al de Buenos Aires. En Facebook se muestra la misma tendencia: mientras la página de $\mathrm{BO}$ posee unas 48 veces menos "me gusta”, publica más del doble de posts que BA. En YouTube las cosas cambian. Buenos Aires actualiza con frecuencia los videos, mientras que el canal de Bolonia está prácticamente inactivo.

Más de la mitad de los contenidos publicados en la cuenta de Twitter de BA en el periodo de registro son informaciones de servicio (30,64\%) y mensajes promocionales (26,81\%) (gráfico 1 ). La actividad de la cuenta de BO se concentra en cuatro categorías: prensa $(21,87 \%)$, servicio $(21,46 \%)$, promocionales $(16,43 \%)$ y agenda del día (11,60\%). En comparación con $\mathrm{BO}$, la cuenta de BA publica un porcentaje significativo de tuits con información de bien público y con contenidos institucionales. La diferencia es importante, además, en la categoría "comunicación política”, que para BA representa un 7,23\% del total de tuits, contra el 0,41 $\%$ de BO. La cuenta de BO, por su parte, está fuertemente dedicada a la información de prensa y a la difusión de la agenda del día, con cerca de un tercio del total de tuits, que en BA representan solo el 4,26\%. BO se distingue, también, por publicar con frecuencia contenidos compartidos por usuarios: el 8,42 \% de los tuits corresponden a la categoría "UGC", mientras que en BA no se registra este tipo de contenidos. También se diferencia, en menor medida, por ofrecer contenidos de gobierno abierto: el 3,49\% contra el 0,85 \% de BA. Si bien los valores resultan marginales, cabe señalar que $\mathrm{BO}$ destina un porcentaje de su actividad a las respuestas públicas a usuarios y a iniciativas de crowdsourcing, mientras que BA no registra este tipo de contenidos.

Al igual que en Twitter, el uso de la página de Facebook de BA está concentrado en contenidos de servicio $(28,71 \%)$ y promocionales $(21,78 \%)$ (gráfico 2). La mayor diferencia se observa en la comunicación política, 


\section{Gráfico 1 \\ Porcentaje de publicaciones en Twitter según categoría de contenidos en el total del periodo}

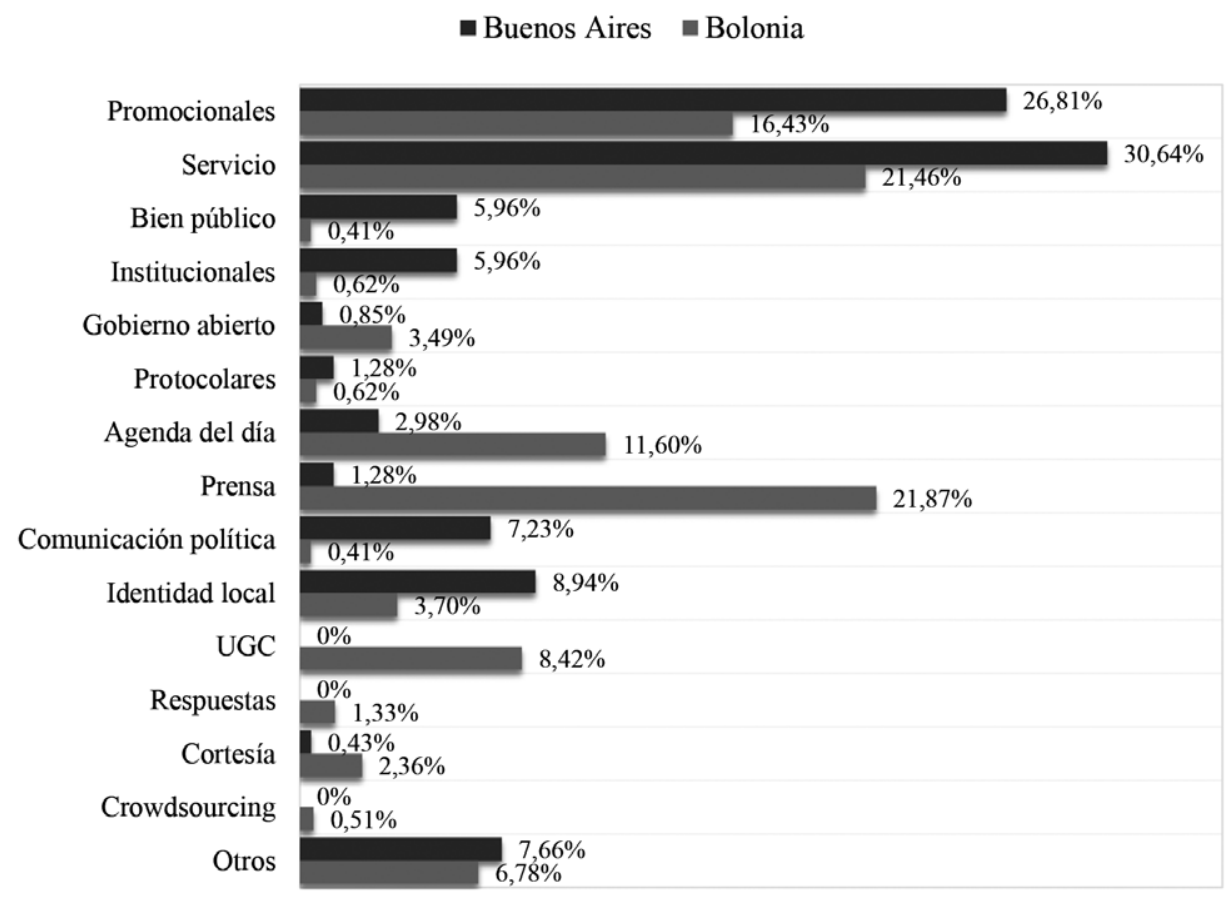

Fuente: elaboración propia.

que en esta red social representa un 15,84 \% de los posts, en comparación con el 7,23\% del total de tuits en la cuenta oficial de la ciudad. La página oficial de $\mathrm{BO}$ conserva las publicaciones de servicio y promocionales, con $32,14 \%$ y $18,30 \%$ respectivamente, al tope de su actividad. Sin embargo, los contenidos generados por los usuarios (UGC) (14,73\%) superan la difusión de la agenda del día (9,82\%) y la información de prensa $(7,14 \%)$. La comparación entre ambas ciudades en el resto de las categorías sigue la tendencia de Twitter: BA refuerza su identidad local y prioriza la información institucional y de bien público; mientras que $\mathrm{BO}$ concede mayor espacio al gobierno abierto y al involucramiento con los usuarios a través de saludos de cortesía. Ninguna de las administraciones públicas registra publicaciones que alienten a la colaboración (crowdsourcing) en el periodo considerado. 


\section{Gráfico 2 \\ Porcentaje de publicaciones en Facebook según categoría de contenidos en el total del periodo*}

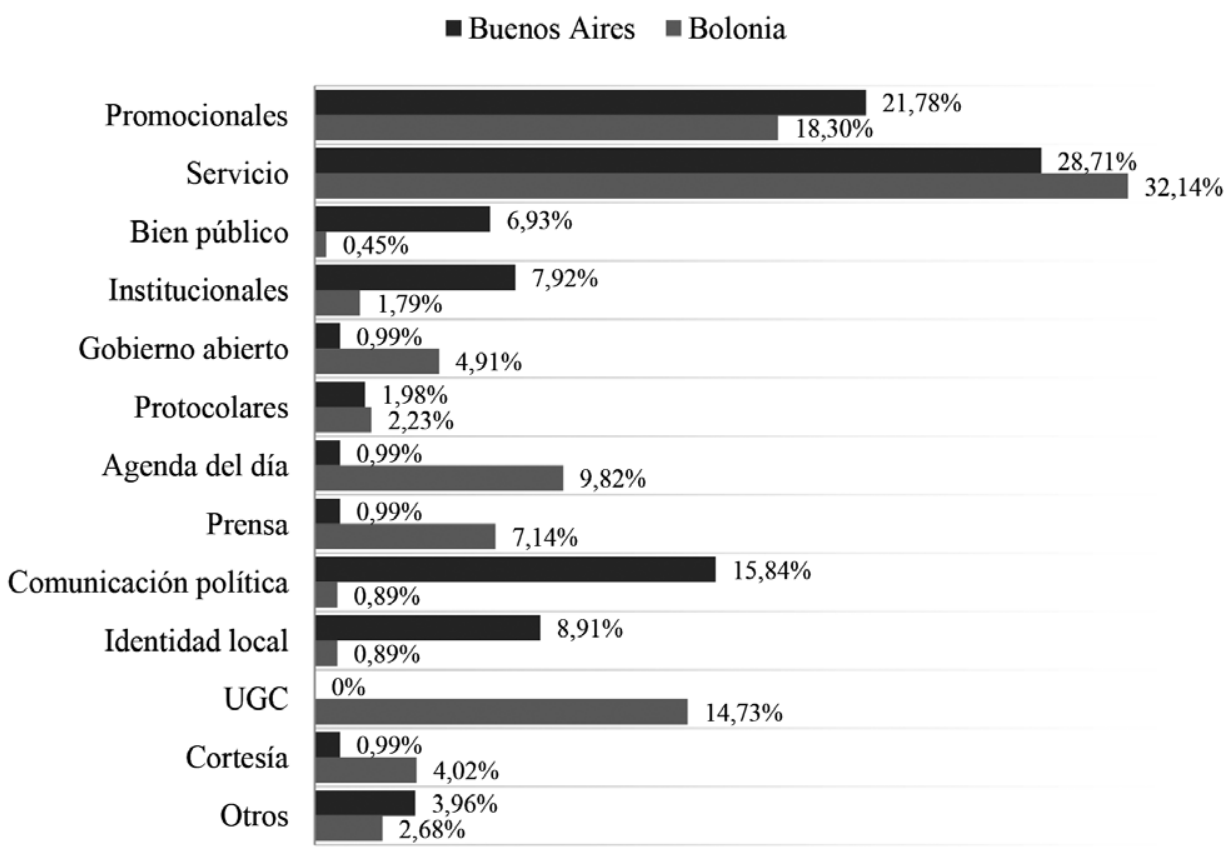

* Se representan solo las categorías que registran valores para alguna de las ciudades consideradas.

Fuente: elaboración propia.

En el caso de los canales de YouTube, la comparación entre ambas ciudades no resulta relevante, ya que el de $\mathrm{BO}$ permaneció prácticamente inactivo (en las seis semanas de registro solo subió un video nuevo). Lo interesante, en cambio, es observar la diferencia en el uso prioritario que hace BA de esta red social respecto de sus cuentas en Twitter y Facebook. El canal de YouTube de BA publica contenido político, orientado a la promoción de la gestión de los funcionarios de gobierno, en casi uno de cada dos videos. Así, mientras que en Twitter y Facebook los contenidos de servicio son los más difundidos, en YouTube este lugar lo ocupa la comunicación política: se publican dos videos de este tipo de comunicación por cada video con contenido promocional, y tres y medio por cada video de servicio. 


\section{Gráfico 3 \\ Porcentaje de publicaciones en YouTube según categoría de contenidos en el total del periodo*}

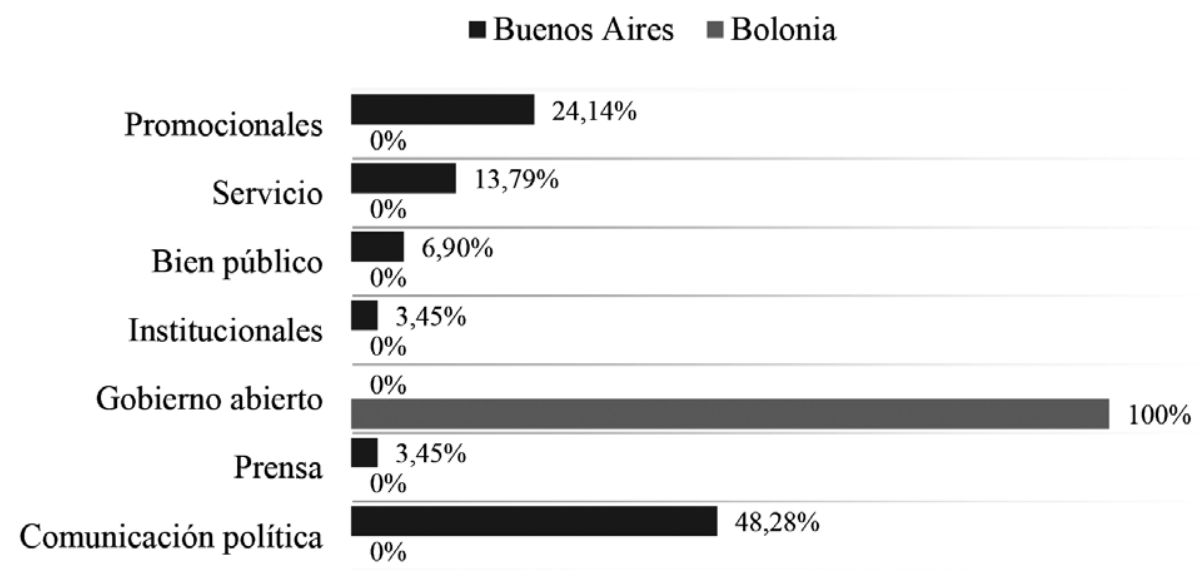

Fuente: elaboración propia.

*Se representan solo las categorías que registran valores para alguna de las ciudades consideradas.

\section{Conclusiones}

Las posibilidades de intercambio con la administración pública y lo que los ciudadanos son capaces de hacer a partir de los contenidos publicados difieren en las dos ciudades comparadas en la investigación. El contrato comunicativo que propone la Ciudad de Buenos Aires es netamente unidireccional, en el que domina el "decir". La escucha se lleva a cabo a través del monitoreo de las búsquedas en la web y los comentarios de los usuarios en las redes, así como también del nivel de repercusión de los posts. Sin embargo, no se materializa en la respuesta directa ni en la amplificación de lo que los ciudadanos hacen y expresan. El contrato de la comuna de Bolonia en las redes sociales es más bien mixto, con preponderancia de mensajes unidireccionales, pero con importantes elementos conversacionales, como la respuesta y la mención a individuos, o la publicación de logros o actividades promovidas por organizaciones privadas o no gubernamentales.

De todos modos, la marcada tendencia hacia la unidireccionalidad no implica necesariamente una falta de reconocimiento y empoderamiento de los usuarios en calidad de ciudadanos. Solo en los contenidos orientados 
a la campaña política permanente de los funcionarios de gobierno, por el hecho de estar centrados en el interés del productor del mensaje, que busca persuadir a los ciudadanos de las bondades de la gestión, se observa un tratamiento de los ciudadanos como target electoral. Tampoco se empodera a los usuarios a través de los contenidos cerrados, que únicamente difunden informaciones sobre las cuales ya no cabe lugar para la participación, lo cual ocurre con los mensajes de prensa y, parcialmente, con la agenda del día. No obstante, los posts de servicio, los promocionales, las campañas de bien público, los saludos protocolares, las referencias a la identidad local, los difusión institucional de normas, derechos y planes, y el acceso a datos de gobierno, contribuyen al empoderamiento del ciudadano. Son mensajes abiertos, que alientan la participación y el compromiso con la comunidad, aun cuando resulten, desde un punto de vista lingüístico, simples actos de habla pronunciados en una sola dirección que no generan reciprocidad de intercambio.

A la inversa, los elementos conversacionales de la propuesta comunicativa de la administración pública no implican necesariamente colaboración, sino que pueden circunscribirse a la facilitación de la expresión de ideas, opiniones, intereses y acciones de los ciudadanos. Si bien esta función es fundamental para la consolidación del ideal de una democracia deliberativa, en la que todos los miembros de la comunidad política deben gozar y ejercer el derecho a la libertad de expresión, no alcanza el requerimiento de la puesta en común de recursos para afrontar, colaborativamente, los asuntos de interés general. Publicar contenido generado por los usuarios (UGC), responder a sus inquietudes, mencionarlos y agradecerles por el aporte al bien de la comunidad, o compartir sus experiencias personales, constituyen acciones de apertura que, sin embargo, no ponen en marcha el paso del e-government al we-government. En efecto, las iniciativas de crowdsourcing en las redes sociales son marginales en los dos casos considerados en la investigación.

Por un lado, el modelo de gestión de las redes sociales es más abierto e inclusivo en Buenos Aires que en Bolonia y, por otro lado, más cerrado y autorreferencial. Las características "abierto" o "cerrado" dependen, aquí, de aquello que los usuarios pueden hacer a partir del acceso a los posts y 
del conjunto de usuarios incluidos en la propuesta comunicativa. Las publicaciones en las cuentas oficiales de Buenos Aires se caracterizan por ser simples, de fácil lectura y comprensión. La información en cada post tiene sentido en sí misma y los hipervínculos conducen a publicaciones que amplían la información ya brindada. Si se trata de información clave, el post se repite en distintos horarios, lo que revela que se apunta a seguidores ocasionales, que no acceden a las redes diariamente o que lo hacen solo durante algún momento del día. Estas dos particularidades configuran un uso de corte masivo de las redes, bajo la lógica broadcast de difusión "de uno a muchos". Si bien este modelo no respeta la cultura conversacional de las redes, corre con la ventaja de la inclusión de los usuarios ocasionales y con menores competencias digitales. En contrapartida, las cuentas son fuertemente autorreferenciales: mencionan casi exclusivamente otras cuentas oficiales del Gobierno de la Ciudad de Buenos Aires, incluyen hipervínculos que remiten a su propio sitio web e insertan logos oficiales a las imágenes, los cuales emplean el mismo color amarillo que identifica al partido que gobierna actualmente la ciudad. Si bien estas prácticas no contribuyen a la conversación pública, resultan adecuadas si lo que se busca es reforzar la identidad de la administración pública en contextos de debilidad institucional o ante la decisión estratégica de potenciar la marca ciudad.

Las cuentas oficiales de Bolonia poseen, en cambio, una gramática más rebuscada. La voluntad de entrar en conversación con los usuarios, asumiendo el lenguaje propio de las redes, conlleva una mayor complejidad. En ocasiones sobreutiliza menciones con arroba, etiquetas con hashtag o con la forma de "[...]" para orientar la lectura. En ciertos casos se hace necesario acceder a los hipervínculos incluidos en los posts para comprender la información brindada, lo cual da la pauta de que apunta a seguidores habituales y consolidados, con competencias adquiridas en el manejo del lenguaje y las dinámicas interactivas de las redes, que ingresan periódicamente y son capaces de descifrar el contenido de las etiquetas. Si bien estas características demuestran que Bolonia logró adoptar la lógica conversacional de las redes, lo que la sitúa a la vanguardia en términos de facilitación de la expresión de los ciudadanos y organizaciones, surge la pregunta de si el resultado final no es más que el refuerzo de un público de nicho, dejando fuera a aquellos usuarios que están interesados pero son menos expertos 
en el uso de las redes. La cuestión se plantea sobre todo en Twitter, por haber desarrollado una gramática propia y por ser la más adecuada para la difusión de información de manera inmediata. Twitter es la plataforma que registra mayor nivel de actividad en redes en ambas ciudades y, sin embargo, está todavía poco difundida entre los ciudadanos argentinos e italianos en comparación con Facebook.

La evaluación de las buenas prácticas de la administración pública en las redes sociales parece estar atada al contexto. ¿Es preferible un modelo que incluye a un número vasto de usuarios o uno que dialoga con ellos de manera personalizada? ¿Resulta oportuno proteger la identidad institucional o difundir ideas y acciones de terceros, y ofrecerse incluso como espacio para la generación de relaciones entre estos y los usuarios? A paridad en la infraestructura de acceso a Internet, en el nivel de penetración de las redes y en el tiempo que los usuarios permanecen en ellas, la respuesta a estos cuestionamientos depende de la solidez de la imagen institucional de la administración pública, así como también de la dimensión de su jurisdicción en términos de número de habitantes a los que brinda su servicio. Un contexto que requiere promover el conocimiento del ente local entre los ciudadanos y potenciar su confianza en él obliga a ensayar estrategias diferentes a aquellas para territorios en los cuales se respeta la separación entre estado y gobierno y en los que las instituciones públicas gozan de buen prestigio en la opinión pública.

El grado de aprovechamiento de la administración pública de las posibilidades de colaboración con los ciudadanos en las redes sociales, en los dos casos analizados, es marginal. La pregunta clave es, precisamente, si esta situación podrá cambiar o si, por el contrario, es razonable esperar que se mantenga a futuro. Si bien parece fundamental que la administración pública ingrese a la conversación pública de las redes, es probable que los mayores esfuerzos sigan estando dedicados a la publicación de contenidos acompañados de prácticas unidireccionales. La administración pública tiene la obligación de acercarse al ciudadano y, en la medida en que continúe consolidándose la tendencia a pasar más tiempo en redes sociales que en la visita a sitios web de las instituciones, la información institucional, de prensa o servicio, deberá tener presencia también allí. 
Se podrá exigir a las administraciones públicas que la colaboración con los usuarios-ciudadanos gane posiciones en el conjunto de su propuesta comunicativa. Sin embargo, la diseminación y la simple conversación constituirán probablemente una parte significativa del contrato ofrecido. Si esto es así, las futuras investigaciones deberían pasar de la descripción de lo que las administraciones hacen en las redes sociales al análisis de lo que generan con lo que hacen, esto es, focalizarse en la relación entre la práctica comunicativa y los eventuales resultados en términos de reconocimiento y empoderamiento de los usuarios en tanto ciudadanos.

\section{Referencias}

Arata, G. (2014). Enti local e social network. Il Mulino, 2, 323-328.

Balcells, J., Padró-Solanet, A. y Serrano, I. (2013). Twitter en los ayuntamientos catalanes: una evaluación empírica de usos y percepciones. En Criado, J. I. y Rojas Martín, F. (eds.), Las redes sociales digitales en la gestión y las políticas públicas (pp. 62-81). Barcelona: Escola d'Administració Pública de Catalunya. Recuperado el 10 de marzo de 2015 de http:/ / eapc.gencat.cat/web/.content/home/publicacions/col_leccio_obres_digitals/15_redes_sociales/od_15_las_ redes_sociales.pdf

Bonsón, E., Torres, L., Royo, S. y Flores, F. (2012). Local e-government 2.0: Social media and corporate transparency in municipalities. Government Information Quarterly, 29, 123-132.

Cencis/Ucsi (2013). $11^{\circ}$ Rapporto Censis/Ucsi sulla comunicazione. Recuperado el 10 de marzo de 2015 de http:/ / www.censis.it/ 17? shadow_ pubblicazione $=120563$

Cippec (2014). Gobierno electrónico local: Índice Nacional de Páginas Web Municipales. Recuperado el 10 de marzo de 2015 de http:/ / www. cippec.org/documents/10179/51827/126+DT+DL,\%20Gobier no+electrónico+local+indice+nacional+de+paginas+web+ mu- 
nicipales+2014,\%20Schejtman+Calamari+Adur).pdf/fab14bf74793-4533-947d-52febc94fdd 1

Cogo, G. (2012). I social network nella PA. Santarcangelo di Romagna: Maggioli.

ComScore (2013). Futuro Digital Argentina 2013. Recuperado el 10 de marzo de 2015 de http://www.comscore.com/lat/Insights/Presentations-and-Whitepapers/2013/2013-Argentina-DigitalFuture-in-Focus

ComScore (2014). Futuro Digital Argentina 2014. Recuperado el 10 de marzo de 2015 de http://www.comscore.com/lat/Insights/Presentations-and-Whitepapers/2014/2014-Argentina-DigitalFuture-in-Focus

Criado, I. (2011). Redes sociales digitales y administraciones públicas: ¿Hacia una nueva manera de relación con la ciudadanía? Telos, 89, 1-4. Recuperado el 10 de marzo de 2015 de http://telos.fundaciontelefonica.com/url-direct/pdf-generator?tipoContenido=articuloT elos\&idContenido $=2011102417130001$ \&idioma $=\mathrm{es}$

Criado, J. I. y Rojas Martín, F. (2013). Aproximación general sobre la adopción y uso de las redes sociales en las administraciones públicas. En Criado, J. I. y Rojas Martín, F. (eds.), Las redes sociales digitales en la gestión y las políticas públicas (pp. 16-31). Barcelona: Escola d’Administració Pública de Catalunya. Recuperado el 10 de marzo de 2015 de http://eapc.gencat.cat/web/.content/home/publicacions/col_leccio_obres_digitals/15_redes_sociales/od_15_las_ redes_sociales.pdf

Generalidad de Cataluña-España (2010). Guía de uso y estilo en redes sociales. Recuperado el 10 de marzo de 2015 de http: / / www.gencat.cat/ web/meugencat/documents/guia_usos_xarxa_es.pdf

Gobierno Vasco-España (2011). Guía de uso y estilo en las redes sociales. Recuperado el 10 de marzo de 2015 de http:/ / www.irekia.euska- 
di.net/es/news/6717-guia-usos-estilo-las-redes-sociales-del-gobierno-vasco

Hofmann, S., Beverungen, D., Räckers, M.y Becker, J. (2013). What makes local governments' online communications successful? Insights form a multi-method analysis of Facebook. Government Information Quarterly, 30, 387-396.

Lizette Martínez, V. (2011). Ayuntamientos en Facebook: ¿El tablón de anuncios del siglo XXI? Telos, 89, 1-11. Recuperado el 10 de marzo de 2015 de http://telos.fundaciontelefonica.com/url-direct/ pdf-generator?tipoContenido $=$ articuloTelos\&idContenido $=201$ 1102412450001 \&idioma $=$ es

Maultasch de Oliveira, G. y Welch, E. (2013). Social media use in local government: Linkage of technology, task, and organizational context. Government Information Quarterly, 30, 397-405.

Mergel, I. (2013). A framework for interpreting social media interactions in the public sector. Government Information Quarterly, 30, 327-334.

Mergel, I. y Bretschneider, S. I. (2013). A three-stage adoption process for social media use in government. Public Administration Review, 3, 390-400.

Ministerio para la Administración Pública y la Innovación-Italia (2011). Vademecum Pubblica Amministrazione e social media. Recuperado el 10 de marzo de 2015 de http:/ /www.funzionepubblica.gov.it/ media/982042/vademecum_pubblica_amministrazione_e_social_media.pdf

Montanari, F., Pattaro, A. F. y Scapolan, A. (2013). Comuni 2.0. Un'indagine esplorativa sull'utilizzo dei social media nei Comuni italiani di medie e grandi dimensioni. Azienda Pubblica, 2, 191-220. 
Mossberger, K., Wu, Y. y Crawford, J. (2013). Connecting citizens and local governments? Social media and interactivity in major U.S. cities. Government Information Quarterly, 30, 351-358.

Osimo, D. y Punie, Y. (2008). Las redes sociales y la administración pública: oportunidades y retos. Bit, 17, 73-77.

Pereyra Caramé, T. (2012). Las administraciones públicas en las redes sociales. Anuario de la Facultad de Derecho - Universidad de Alcalá $V, 449-474$.

Pew Research Center (2015). Social Media Update 2013. Recuperado el 10 de marzo de 2015 de http: / / www.pewinternet.org/files/2013/12/ PIP_Social-Networking-2013.pdf

Secretaría de Comunicación Social-Brasil (2013). Manual de orientación para participar en redes sociales. Recuperado el 10 de marzo de 2015 de http://publications.iadb.org/bitstream/handle/11319/3509/ Manual\%20de\%20orientaci\%C3\%B3n\%20para\%20participar\%20 en $\% 20$ redes $\% 20$ sociales.pdf ? sequence $=1$

Secretaría de Cultura de la Nación (2013). Encuesta Nacional de Consumos Culturales. Recuperado el 10 de marzo de 2015, de: http://sinca. cultura.gob.ar/sic/publicaciones/libros/EECC.pdf

Sixto García, J. (2012). Uso de las redes sociales en la Administración pública gallega: ¿una técnica de marketing 2.0? Pensar la Publicidad, 2, 345-363.

Ure, M. y Parselis, M. (2013). Argentine Media and Journalists Enhancing and Polluting of Communication on Twitter. International Journal of Communication, 7, Feature 1784-1800. Recuperado el 10 de marzo de 2015 de http://ijoc.org/index.php/ijoc/article/ view/2178/970

We Are Social (2015). Digital, Social \& Mobile WorldWide 2015. Recuperado el 10 de marzo de 2015 de http://wearesocial.net/ blog/2015/01/digital-social-mobile-worldwide-2015/ 